preprint SHEP-06-01

July 12, 2018

\title{
Di-photon Higgs signals at the LHC in the Next-to-Minimal Supersymmetric Standard Model
}

\author{
Stefano Moretti and Shoaib Munir \\ High Energy Physics Group, School of Physics 83 Astronomy, \\ University of Southampton, Southampton, SO17 1BJ, UK
}

\begin{abstract}
The NMSSM contains a Higgs singlet in addition to the two Higgs doublets typical of the MSSM, thus resulting in a total of seven physical Higgs mass states. Therefore, the phenomenology of the NMSSM Higgs sector can vary considerably from that of the MSSM and there are good prospects of finding in regions of the NMSSM parameter space Higgs signals that cannot be reproduced in the MSSM. We examined here the two-photon decay mode of a Higgs boson and found that up to three neutral Higgs states, heavy and/or light, could be simultaneously observable at the LHC, a possibility precluded to the MSSM. There are also some possibilities that only the lightest NMSSM Higgs boson be detectable via this mode, with a mass beyond the upper limit of the corresponding MSSM state, thus also allowing to distinguish between the two scenarios. However, in most of the NMSSM parameter space the configurations of the non-minimal model are not very different from those arising in the minimal case.
\end{abstract}

\section{$1 \quad$ Introduction}

The Minimal Supersymmetric Standard Model (MSSM) [1] is affected by the so-called ' $\mu$ problem'. The MSSM Superpotential can be written as [2]

$$
W_{\mathrm{MSSM}}=\hat{Q} \hat{H}_{u} \mathbf{h}_{\mathbf{u}} \hat{U}^{C}+\hat{H}_{d} \hat{Q} \mathbf{h}_{\mathbf{d}} \hat{D}^{C}+\hat{H}_{d} \hat{L} \mathbf{h}_{\mathbf{e}} \hat{E}^{C}+\mu \hat{H}_{u} \hat{H}_{d}
$$

(Hereafter, hatted variables describe Superfields while un-hatted ones stand for the corresponding scalar Superfield components). The last term in the above equation contains a dimensionful parameter, $\mu$. Upon Electro-Weak Symmetry Breaking (EWSB), it provides a 
contribution to the masses of both Higgs bosons and Higgsino fermions. Furthermore, the associated soft Supersymmetry (SUSY) breaking term $B \mu H_{u} H_{d}$ mixes the two Higgs doublets. Now, the presence of $\mu$ in the Superpotential before EWSB indicates that its natural value would be either 0 or the Planck mass $M_{P}$. On the one hand, $\mu=0$ would mean no mixing is actually generated between Higgs doublets at any scale and the minimum of the Higgs potential occurs for $\left\langle H_{d}\right\rangle=0$, so that one would have in turn massless downtype fermions and leptons after $\mathrm{SU}(2)$ symmetry breaking. On the other hand, $\mu \approx M_{P}$ would reintroduce a 'fine-tuning problem' in the MSSM since the Higgs scalars would acquire a huge contribution $\sim \mu^{2}$ to their squared masses (thus spoiling the effects of SUSY, which effectively removes otherwise quadratically divergent contributions to the Higgs mass from SM particles). Therefore, the values of this (theoretically arbitrary) parameter $\mu$ are phenomenologically constrained to be close to $M_{\text {SUSY }}$ or $M_{W}[3$.

The most elegant solution to the $\mu$-problem is to introduce a new singlet Higgs field $S$ and replace $B \mu H_{u} H_{d}$ by an interaction $\sim S\left(H_{u} H_{d}\right)$. When the extra scalar field $S$ acquires a Vacuum Expectation Value (VEV), an effective $\mu$ term, naturally of the EW scale, is generated automatically. This idea has been implemented in the Next-to-Minimal Supersymmetric Standard Model (NMSSM) 4], described by the Superpotential

$$
W_{\mathrm{NMSSM}}=\hat{Q} \hat{H}_{u} \mathbf{h}_{\mathbf{u}} \hat{U}^{C}+\hat{H}_{d} \hat{Q} \mathbf{h}_{\mathbf{d}} \hat{D}^{C}+\hat{H}_{d} \hat{L} \mathbf{h}_{\mathbf{e}} \hat{E}^{C}+\lambda \hat{S}\left(\hat{H}_{u} \hat{H}_{d}\right)+\frac{1}{3} \kappa \hat{S}^{3}
$$

where $\hat{S}$ is an extra Higgs iso-singlet Superfield, $\lambda$ and $\kappa$ are dimensionless couplings and the last $\left(Z_{3}\right.$ invariant) term is required to explicitly break the dangerous Peccei-Quinn (PQ) U(1) symmetry [5] $]^{1}$. (See Ref. [7] for NMSSM Higgs sector phenomenology with an exact or slightly broken PQ symmetry.) Furthermore, a $Z_{2}^{R}$ symmetry can be imposed to avoid the so-called 'domain-walls problem' of the NMSSM 8], through harmless tadpoles breaking the global $Z_{3}$ symmetry 9]. (Alternative formulations - known as the Minimal Non-minimal Supersymmetric Standard Model (MNSSM) and new Minimally-extended Supersymmetric Standard Model (nMSSM) - vetoing the presence of the $\sim \kappa \hat{S}^{3}$ term and allowing instead suitable linear tadpole terms through the enforcement of discrete $R$-symmetries - also exist [10.) Another positive feature of all these non-minimal SUSY models is that they predict the existence of a (quasi-)stable singlet-type neutralino (the singlino) that could be responsible for the Dark Matter (DM) of the universe 11. Finally, notice that, in these extended SUSY models, the singlet Superfield $\hat{S}$ has no SM gauge group charge (so that MSSM gauge coupling unification is preserved) and that one can comfortably explain the baryon asymmetry of the Universe by means of a strong first order EW phase transition [12 (unlike the MSSM, which would require a light top squark and Higgs boson barely compatible with current experimental bounds [13]).

Clearly, in eq. (2), upon EWSB a VEV will be generated for the real scalar component of $\hat{S}$ (the singlet Higgs field), $\langle S\rangle$, alongside those of the two doublets $\left\langle H_{u}\right\rangle$ and $\left\langle H_{d}\right\rangle$ (related by the parameter $\tan \beta=<H_{u}>/<H_{d}>$ ). In the absence of fine-tuning, one should expect these three VEVs to be of the order of $M_{\mathrm{SUSY}}$ or $M_{W}$, so that now one has an

\footnotetext{
${ }^{1}$ One could also gauge the $\mathrm{U}(1)_{\mathrm{PQ}}$ group, so that the $Z_{3}$ symmetry is embedded in the local gauge symmetry [6].
} 
'effective $\mu$-parameter'

$$
\mu_{\mathrm{eff}}=\lambda<S>,
$$

of the required size, thus effectively solving the $\mu$-problem.

\section{Higgs Phenomenology in the NMSSM}

In the NMSSM, the soft SUSY-breaking Higgs sector is described by the Lagrangian contribution

$$
V_{\mathrm{NMSSM}}=m_{H_{u}}^{2}\left|H_{u}\right|^{2}+m_{H_{d}}^{2}\left|H_{d}\right|^{2}+m_{S}^{2}|S|^{2}+\left(\lambda A_{\lambda} S H_{u} H_{d}+\frac{1}{3} \kappa A_{\kappa} S^{3}+\text { h.c. }\right),
$$

where $A_{\lambda}$ and $A_{\kappa}$ are dimensionful parameters of order $M_{\mathrm{SUSY}}$.

As a result of the introduction of an extra complex singlet scalar field, which only couples to the two MSSM-type Higgs doublets, the Higgs sector of the NMSSM comprises of a total of seven mass eigenstates: a charged pair $h^{ \pm}$, three CP-even Higgses $h_{1,2,3}\left(m_{h_{1}}<m_{h_{2}}<m_{h_{3}}\right)$ and two CP-odd Higgses $a_{1,2}\left(m_{a_{1}}<m_{a_{2}}\right)$. Consequently, Higgs phenomenology in the NMSSM may be plausibly different from that of the MSSM and extremely rich of new signals.

For a start, the mass expressions for the CP-even Higgses in the NMSSM can be translated into a modified upper bound on the lightest Higgs mass, $m_{h_{1}}$, as [14]

$$
m_{h_{1}}^{2} \leq \min \left\{m_{Z}^{2}, \frac{1}{2} \kappa<S>\left(4 \kappa<S>+\sqrt{2} A_{\kappa}\right)\right\} .
$$

As the higher order corrections are similar to those in the MSSM, the upper bound on the lightest Higgs boson is different in the NMSSM, reaching 135-140 GeV, for maximal stop mixing and $\tan \beta=2$ [15, 16] (a configuration indeed excluded in the MSSM by LEP data). More in general, the 'little fine tuning problem', resulting in LEP failing to detect a light CPeven Higgs boson, predicted over most of the MSSM parameter space, is much attenuated in the NMSSM, because the mixing among more numerous CP-even or CP-odd Higgs fields enables light mass states being produced at LEP yet they can remain undetected because of their reduced couplings to $Z$ bosons [16].

As for future machines, chiefly the CERN Large Hadron Collider (LHC), quite some work has been dedicated to probing the NMSSM Higgs sector over recent years. Primarily, there have been attempts to extend the so-called 'No-lose theorem' of the MSSM - stating that at least one MSSM Higgs boson should be observed via the usual SM-like production and decay channels at the LHC throughout the entire MSSM parameter space [17] - to the case of the NMSSM [18. From this perspective, it was realised that at least one NMSSM Higgs boson should remain observable at the LHC over the NMSSM parameter space that does not allow any Higgs-to-Higgs decay. However, when the only light non-singlet (and, therefore, potentially visible) CP-even Higgs boson, $h_{1}$ or $h_{2}$, decays mainly to two very light CP-odd Higgs bosons, $h \rightarrow a_{1} a_{1}$, one may not have a Higgs signal of statistical significance at the LHC [19. In fact, further violations to the theorem may well occur if one enables Higgs-to-SUSY decays (e.g., into neutralino pairs, yielding invisible Higgs signals). 
While the jury is still out on whether a 'No-lose theorem' can be proved for the NMSSM, we are here concerned with an orthogonal approach. We asked ourselves if a, so to say, 'Moreto-gain theorem' can be formulated in the NMSSM. That is, whether there exist regions of the NMSSM parameter space where more Higgs states of the NMSSM are visible at the LHC than those available within the MSSM. In our attempt to overview all such possibilities, we start by considering here the case of the di-photon decay channel of a neutral Higgs boson. This mode can be successfully probed in the MSSM, but limitedly to the case of one Higgs boson only, which is CP-even and rather light. We will show that in the NMSSM one can instead potentially have up to three di-photon signals of Higgs bosons, involving not only CP-even but also CP-odd states, the latter with masses up to $600 \mathrm{GeV}$ or so. In fact, even when only one di-photon signal can be extracted in the NMSSM, this may well be other than the $h_{1}$ state. When only the latter is visible, finally, it can happen that its mass is larger than the maximum value achievable within the MSSM. In all such cases then, the existence of a non-minimal SUSY Higgs sector would be manifest.

\section{Parameter Space Scan}

The choice of parameter space largely depends on the version of NMSSM under consideration, or more specifically, on the implication of unification of parameters at some very high scale. This, in turn, leans on the technique adopted for the breaking of SUSY, since it still remains undetected in nature. The advantage of assuming unification of masses and couplings in a SUSY model is twofold. Firstly, it enormously reduces the labour from a phenomenological perspective by minimising the number of parameters required to extrapolate physical information from the model. Secondly, and theoretically more crucially, it caters to the fundamental objective of constructing a Grand Unification Theory (GUT), which was, to a great extent, responsible for devising SUSY in the first place.

A particular case of a low energy NMSSM with gravity mediated SUSY breaking was studied in 20]. As usual, in such a model, SUSY is assumed to be broken in some hidden sector and then mediated to the physical sector through gravitational interactions. Such a model implies unification of the couplings and soft masses at the GUT scale, which are then run down, using renormalisation group equations, to the weak scale or to some other scale at which the theory is being tested. This results in only a handful of parameters to deal with.

For a more general study of the NMSSM Higgs sector with a wider range of parameters, we used here the publicly available fortran code NMHDECAY (version 1.1) developed in Ref. [21]. This program computes the masses, couplings and decay widths of all the Higgs bosons of the NMSSM in terms of its parameters at the EW scale. For our purpose, instead of postulating unification, and without taking into account the SUSY breaking mechanism, we fixed the soft SUSY breaking terms to a very high value, so that they have little or no contribution to the outputs of the parameter scans. Consequently, we are left with six free parameters.

Our parameter space includes the Yukawa couplings $\lambda$ and $\kappa$, the soft trilinear terms $A_{\lambda}$ and $A_{\kappa}$, plus $\tan \beta$ and $\mu_{\text {eff }}=\lambda\langle S\rangle$. The computation of the spectrum includes leading two loop terms, EW corrections and propagator corrections. The decay widths, however, do not 
include three body decays. The NMHDECAY program also takes into account theoretical as well as experimental constraints from negative Higgs searches at LEP, along with the unconventional channels relevant for the NMSSM.

We have used the NMHDECAY code to scan over the NMSSM parameter space defined through the aforementioned six parameters taken in the following intervals:

$$
\begin{gathered}
\lambda: 0.0001-0.75, \quad \kappa:-0.65-+0.65, \quad \tan \beta: 1.6-54 \\
\mu, A_{\lambda}, A_{\kappa}:-1000-+1000 \mathrm{GeV} .
\end{gathered}
$$

Remaining soft terms which are fixed in the scan include:

- $m_{Q_{3}}=m_{U_{3}}=m_{D_{3}}=m_{L_{3}}=m_{E_{3}}=2 \mathrm{TeV}$,

- $A_{U_{3}}=A_{D_{3}}=A_{E_{3}}=1.5 \mathrm{TeV}$,

- $m_{Q}=m_{U}=m_{D}=m_{L}=m_{E}=2 \mathrm{TeV}$,

- $M_{1}=M_{2}=M_{3}=3 \mathrm{TeV}$.

In line with the assumptions made in [22, the allowed decay modes for neutral NMSSM Higgs bosons are ${ }^{2}$ :

$$
\begin{array}{cll}
h, a \rightarrow g g, \quad h, a \rightarrow \mu^{+} \mu^{-}, & h, a \rightarrow \tau^{+} \tau^{-}, & h, a \rightarrow b \bar{b}, \quad h, a \rightarrow t \bar{t}, \\
h, a \rightarrow s \bar{s}, \quad h, a \rightarrow c \bar{c}, & h \rightarrow W^{+} W^{-}, \quad h \rightarrow Z Z, \\
h, a \rightarrow \gamma \gamma, \quad h, a \rightarrow Z \gamma, \quad h, a \rightarrow \text { Higgses }, \quad h, a \rightarrow \text { sparticles } .
\end{array}
$$

(Notice that for the pseudoscalar Higgses, the decay into vector boson pairs is not allowed due to CP-conservation.) Here, 'Higgses' refers to any final state involving all possible combination of two Higgs bosons (neutral and/or charged) or of one Higgs boson and a gauge vector.

The aforementioned range of parameters is borrowed from Ref. [22], where the authors singled out the most difficult scenarios for NMSSM Higgs discovery at the LHC (using the mentioned code). In this paper they also concluded, as a follow up of the NMSSM 'NoLose theorem', that the region where no Higgs is observable, due to its decays into the unconventional channel $h \rightarrow a_{1} a_{1}$, comprises of only about $1 \%$ of the entire parameter space of the NMSSM. In short, our idea is to explore the same range of parameter space and look for the 'best case scenarios' where the discovery of one or more Higgses at the LHC is possible above and beyond what is predicted in the MSSM.

We have performed our scan over several millions of randomly selected points in the specified parameter space. The output, as stated earlier, contains masses, Branching Ratios (BRs) and couplings of the NMSSM Higgses, for all the points which are not forbidden by the various constraints. The points which violate the constraints are eliminated. The surviving data points are then used to determine the cross-sections for NMSSM Higgs hadro-production by using an adapted version of the codes described in [23]. As the SUSY mass scales have been arbitrarily set well above the EW one (see above), the NMSSM Higgs production modes exploitable in simulations at the LHC are those involving couplings to heavy ordinary matter

\footnotetext{
${ }^{2}$ Here, we use the label $h(a)$ to signify any of the neutral CP-even(odd) Higgs bosons of the NMSSM.
} 
only, i.e., (hereafter, $V=W^{ \pm}, Z$ and $Q=b, t$ ) for neutral Higgs production (where the last two channels are only allowed for CP-even Higgs production):

$$
\begin{gathered}
g g \rightarrow \text { Higgs (gluon - fusion, via heavy - quark loops) } \\
g g \rightarrow Q \bar{Q} \text { Higgs (heavy - quark associated production), } \\
q q \rightarrow q q V^{*} V^{*} \rightarrow q q \text { Higgs (vector - boson - fusion) } \\
q \bar{q} \rightarrow V \text { Higgs (Higgs - strahlung) }
\end{gathered}
$$

(These are the so-called 'direct' Higgs production modes.) Here, 'Higgs' refers to any possible neutral Higgs boson.

Production and decay rates for NMSSM neutral Higgs bosons have then been multiplied together to yield inclusive event rates, assuming a LHC luminosity of $100 \mathrm{fb}^{-1}$ throughout.

\section{Spectrum Configuration and Inclusive Event Rates}

As an initial step towards the analysis of the data, we computed the total cross-section times BR into $\gamma \gamma$ pairs against each of the six parameters of the NMSSM, for each Higgs boson. We have assumed all production modes described in the above Section and started by computing total (i.e., fully inclusive) rates $^{3}$. We are here focusing on the $\gamma \gamma$ decay mode since it is the most promising channel for the discovery of a (neutral) Higgs boson at the LHC in the moderate Higgs mass range (say, below $130 \mathrm{GeV}$ ). In fact, since the tail of the $\gamma \gamma$ background falls rapidly with increasing invariant mass of the di-photon pair, signal peaks for heavier Higgses could also be visible in addition to (or instead of) the lightest one, although the cross-section for these processes is relatively very low [24, 25]. As the starting point of our numerical study, based on the ATLAS analysis of Ref. [26], we argue that crosssection times BR rates of $10 \mathrm{fb}$ or so are potentially interesting from a phenomenological point of view, in the sense that they may yield visible signal events, the more so the heavier the decaying Higgs state (also because the photon detection efficiency grows with the Higgs mass [26]). (See Ref. [27] for a preliminary account in this respect.)

Tab. 11 recaps the potential observability of one or more NMSSM Higgs states in the di-photon mode at the LHC, under the above assumptions. It is obvious from the table that one light CP-even Higgs should be observable almost throughout the NMSSM parameter space (in line with the findings of Ref. [22]). However, there is also a fair number of points where two Higgses may be visible simultaneously $\left(h_{1}\right.$ and $h_{2}$ or - more rarely $-h_{1}$ and $\left.a_{1}\right)$, while production and decay of the three lightest Higgses $\left(h_{1}, h_{2}\right.$ and $\left.a_{1}\right)$ at the same time, although possible, occurs for only a very small number of points in the parameter space. Furthermore, the percentage of points for which only the second lightest Higgs state is visible is also non-negligible. These last two conditions are clearly specific to the NMSSM, as they are never realised in the MSSM. Furthermore, while the lone detection of the lightest CP-even NMSSM Higgs boson may mimic a similar signal from the corresponding state in

\footnotetext{
${ }^{3}$ After verifying that the bulk of the signal rates is due to gluon-gluon fusion (even at large Higgs masses), we have eventually decided - for simplicity - to limit ourselves to emulate only this channel. Hence, all the results found below suffer from a slight under-estimate of the signal rates.
} 
the MSSM, the reconstructed mass may well be beyond the upper mass limit in the MSSM, this possibility also pointing towards the evidence of a NMSSM Higgs sector. Finally, none of the two heaviest NMSSM neutral Higgs states $\left(h_{3}\right.$ and $\left.a_{2}\right)$ will be visible in the di-photon channel at the LHC (given their large masses).

We have then plotted the cross-sections times BR for the three potentially observable Higgses against the various NMSSM parameters. These plots, shown in Figs. 1-6, reveal that the distribution of possibly visible points (i.e., of those yielding cross-section time BR rates in excess of $10 \mathrm{fb}$ ) is quite homogeneous over the NMSSM parameter space and not located in some specific parameter areas (i.e., in a sense, not 'fine-tuned'). The distribution of the same points as a function of the corresponding Higgs masses can be found in Fig. 7. Of particular relevance is the distribution of points in which only the NMSSM $h_{1}$ state is visible, when its mass is beyond the upper mass limit for the corresponding CP-even MSSM Higgs state, which is shown in Fig. $8^{4}$. This plot reveals that about $93 \%$ of the NMSSM $h_{1}$ masses visible alone are expected to be within 2-3 GeV beyond the MSSM bound, hence the two models would be indistinguishable ${ }^{5}$. Nonetheless, there is a fraction of a percent of such points with $m_{h_{1}}$ values even beyond $125 \mathrm{GeV}$ or so (the higher the mass the smaller the density, though), which should indeed allow one to distinguish between the two models. Moreover, by studying the cross-section times BR of the Higgses when two of them are observable against their respective mass differences, Figs. 9-11, one sees that the former are larger than the typical mass resolution in the di-photon channel, so that the two decaying objects should indeed appear in the data as separate resonances. (We have also verified, though not shown here, that their decay widths are small compared to the detector resolution in $M_{\gamma \gamma}$.)

Next, we have proceeded to a dedicated parton level analysis of signal and background processes, the latter involving both tree-level $q \bar{q} \rightarrow \gamma \gamma$ and one-loop $g g \rightarrow \gamma \gamma$ contributions. We have adopted standard cuts on the two photons [26]: $p_{T}^{\gamma}>25 \mathrm{GeV}$ and $\left|\eta^{\gamma}\right|<2.4$ on transverse energy and pseudorapidity, respectively. As illustrative examples of a possible NMSSM Higgs phenomenology appearing at the LHC in the di-photon channel, we have picked up the following three configurations:

1. $\lambda=0.6554, \kappa=0.2672, \mu=-426.48 \mathrm{GeV}, \tan \beta=2.68, A_{\lambda}=-963.30 \mathrm{GeV}$, $A_{\kappa}=30.48 \mathrm{GeV}$;

2. $\lambda=0.6445, \kappa=0.2714, \mu=-167.82 \mathrm{GeV}, \tan \beta=2.62, A_{\lambda}=-391.16 \mathrm{GeV}$, $A_{\kappa}=50.02 \mathrm{GeV}$;

3. $\lambda=0.4865, \kappa=0.3516, \mu=355.63 \mathrm{GeV}, \tan \beta=2.35, A_{\lambda}=519.72 \mathrm{GeV}, A_{\kappa}=$ $-445.71 \mathrm{GeV}$.

\footnotetext{
${ }^{4}$ Notice that the value obtained for $m_{h_{1}}^{\max }$ from NMHDECAY version 1.1, of $\sim 130 \mathrm{GeV}$, based on the leading two-loop approximations described in [21, is a few GeV lower than the value declared in Sect. 1. Besides, for consistency, we use the value of $120 \mathrm{GeV}$ (obtained at the same level of accuracy) as upper mass limit on the lightest CP-even Higgs boson of the MSSM. (Notice that a slightly modified $m_{h_{1}}^{\max }$ value is obtained for the NMSSM from NMHDECAY version 2.1 28], because of the improved mass approximations with respect to the earlier version of the program adopted here.) Eventually, when the LHC is on line, the exercise that we are proposing can be performed with the then state-of-the-art calculations.

${ }^{5}$ Other than an experimental di-photon mass resolution of $2 \mathrm{GeV}$ or so [26] one should also bear in mind here that the mass bounds in both models come with a theoretical error of comparable size.
} 
The first is representative of the case in which only the NMSSM $h_{1}$ boson is visible, but with mass larger than the MSSM upper limit on the corresponding Higgs state. The second and third refer instead to the case when also the $h_{2}$ or $a_{1}$ state are visible, respectively. The final results are found in Fig. 12. The corresponding mass resonances are clearly visible above the continuum di-photon background and discoverable beyond the $5 \sigma$ level. Indeed, similar situations can be found for each of the combinations listed in Tab. 11 and most of these correspond to phenomenological scenarios which are distinctive of the NMSSM and that cannot be reproduced in the MSSM.

\section{Conclusion}

In summary, we have shown that there exists the possibility of establishing a 'More-to-gain theorem' within the NMSSM, as compared to what is expected in the MSSM, in terms of novel Higgs signals appearing in the di-photon discovery channel which can be ascribed to the former but not to the latter. We have showed this to be the case for a few selected NMSSM parameter points, by performing a proper signal-to-background analysis at the partonic level. However, a similar numerical study can easily be extended to encompass sizable regions of the NMSSM parameter space. While the bulk of the latter is in a configuration degenerate with the MSSM case (as far as di-photon signals at the LHC are concerned), non-negligible areas exist where further phenomenological studies have the potential to unveil a non-minimal nature of the underlying SUSY model.

To this end, NMSSM benchmark scenarios, amenable to experimental investigation in the context of full Monte Carlo (MC) analyses, based on event generation and detector simulation, are currently being devised [29]. Also, un upgraded version of HERWIG 30], suitable for MC event generation in the context of non-minimal SUSY models, is currently being prepared 31.

\section{Acknowledgments}

We acknowledge useful email exchanges with Cyril Hugonie. SM would like to thank the British Council under the 'Alliance: Franco British Partnership Programme 2004 (Project Number: PN 04.051)' for travel support in connection with this research. He is also grateful to Fawzi Boudjema and Genevieve Belanger for their kind hospitality at LAPP-TH.

\section{References}

[1] For reviews see, e.g.: H.E. Haber and G.L. Kane, Phys. Rep. 117 (1985) 75; P. Fayet and S. Ferrara, Phys. Rep. 32 (1977) 249; H.P. Nilles, Phys. Rep. 110 (1984) 1; R. Barbieri, Riv. Nuov. Cim. 11 (1988) 1.

[2] J.F. Gunion and H.E. Haber, Nucl. Phys. B 272 (1986) 1; (E) hep-ph/9301205. 
[3] J.E. Kim and H.P. Nilles, Phys. Lett. B 138 (1984) 150.

[4] P. Fayet, Nucl. Phys. B 90 (1975) 104; Phys. Lett. B 64 (1976) 159; Phys. Lett. B 69 (1977) 489 and Phys. Lett. B 84 (1979) 416; H.P. Nilles, M. Srednicki and D. Wyler, Phys. Lett. B 120 (1983) 346; J.M. Frere, D.R. Jones and S. Raby, Nucl. Phys. B 222 (1983) 11; J.P. Derendinger and C.A. Savoy, Nucl. Phys. B 237 (1984) 307; K. Inoue, A. Komatsu and S. Takeshita, Prog. Theor. Phys 68 (1982) 927; (E) ibid. 70 (1983) 330; M. Dine, W. Fischler and M. Srednicki, Phys. Lett. B 104 (1981) 199; A.I. Veselov, M.I. Vysotsky and K.A. Ter-Martirosian, Sov. Phys. JETP 63 (1986) 489 [Zh. Eksp. Teor. Fiz. 90 (1986) 838]; B.R. Greene and P.J. Miron, Phys. Lett. B 168 (1986) 26; J.R. Ellis, J.F. Gunion, H.E. Haber, L. Roszkowski and F. Zwirner, Phys. Rev. D 39 (1989) 844; M. Drees, Int. J. Mod. Phys. A 4 (1989) 3635; U. Ellwanger, M. Rausch de Traubenberg and C.A. Savoy, Phys. Lett. B 315 (1993) 331; P.N. Pandita, Z. Phys. C 59 (1993) 575; S.F. King and P.L. White, Phys. Rev. D 52 (1995) 4183.

[5] R.D. Peccei and H.R. Quinn, Phys. Rev. Lett. 38 (1977) 1440 and Phys. Rev. D 16 (1977) 1792.

[6] M. Cvetic, D.A. Demir and L. Everett, Phys. Rev. D 56 (1997) 2861; D.A. Demir and L. Everett, Phys. Rev. D 69 (2004) 015008; T. Han, P. Langacker and B. McElrath, Phys. Rev. D 70 (2004) 115006; S.F. King, S. Moretti and R. Nevzorov, Phys. Lett. B 634 (2006) 278, Phys. Rev. D 73 (2006) 035009 and hep-ph/0601269 (and references therein).

[7] D.J. Miller and R. Nevzorov, hep-ph/0309143 and hep-ph/0411275 D.J. Miller, S. Moretti and R. Nevzorov, hep-ph/0501139.

[8] H.P. Nilles, M. Srednicki, and D. Wyler, Phys. Lett. B 124 (1983) 337; A.B. Lahanas, Phys. Lett. B 124 (1983) 341; U. Ellwanger, Phys. Lett. B 133 (1983) 187; J. Bagger and E. Poppitz, Phys. Rev. Lett. 71 (1993) 2380; J. Bagger, E. Poppitz and L. Randall, Nucl. Phys. B 426 (1994) 3; V. Jain, Phys. Lett. B 351 (1995) 481; S.A. Abel, Nucl. Phys. B 480 (1996) 55; C.F. Kolda, S. Pokorski and N. Polonsky, Phys. Rev. Lett. 80 (1998) 5263; S.A. Abel, S. Sarkar and P.L. White, Nucl. Phys. B 454 (1995) 663.

[9] C. Panagiotakopoulos and K. Tamvakis, Phys. Lett. B 446 (1999) 224.

[10] C. Panagiotakopoulos and K. Tamvakis, Phys. Lett. B 469 (1999) 145; C. Panagiotakopoulos and A. Pilaftsis, Phys. Rev. D 63 (2001) 055003; A. Dedes, C. Hugonie, S. Moretti and K. Tamvakis, Phys. Rev. D 63 (2001) 055009

[11] A. Menon, D.E. Morrissey and C.E.M. Wagner, Phys. Rev. D 70 (2004) 035005; V. Barger, P. Langacker and H.-S. Lee, Phys. Lett. B 630 (2005) 85; J.F. Gunion, D. Hooper and B. McElrath, hep/ph0509024; G. Belanger, F. Boudjema, C. Hugonie, A. Pukhov and A. Semenov, JCAP 0509 (2005) 001.

[12] M. Bastero-Gil, C. Hugonie, S.F. King, D.P. Roy and S. Vempati, Phys. Lett. B 489 (2000) 359; S.W. Ham, S.K. Oh, E.J. Yoo, C.M. Kim and D. Son, Phys. Rev. D 70 
(2004) 075001; K. Funakubo, S. Tao and F. Toyoda, Prog. Theor. Phys. 114 (2005) 369.

[13] M. Carena, M. Quiros, M. Seco and C.E.M. Wagner, Nucl. Phys. B 650 (2003) 24; T. Konstandin, T. Prokopec, M.G. Schmidt and M. Seco, Nucl. Phys. B 738 (2006) 1.

[14] D.J. Miller, R. Nevzorov and P.M. Zerwas, Nucl. Phys. B 681 (2004) 3.

[15] M. Masip, R. Muñoz-Tapia and A. Pomarol, Phys. Rev. D 57 (1998) 5340.

[16] U. Ellwanger and C. Hugonie, Eur. Phys. J. C 25 (2002) 297.

[17] J. Dai, J.F. Gunion, R. Vega, Phys. Lett. B 315 (1993) 355 and Phys. Lett. B 345 (1995) 29; J.R. Espinosa, J.F. Gunion, Phys. Rev. Lett. 82 (1999) 1084.

[18] U. Ellwanger, J.F. Gunion and C. Hugonie, hep-ph/0111179; U. Ellwanger, J.F. Gunion, C. Hugonie and S. Moretti, hep-ph/0305109 and hep-ph/0401228; D.J. Miller and S. Moretti, hep-ph/0403137.

[19] D. Zerwas and S. Baffioni, private communication; S. Baffioni, talk presented at "GdR Supersymétrie 2004, 5-7 July 2004, Clermont-Ferrand, France.

[20] C. Hugonie and S. Moretti, hep-ph/0110241.

[21] U. Ellwanger, J.F. Gunion and C. Hugonie, JHEP 0502 (2005) 066.

[22] U. Ellwanger, J.F. Gunion and C. Hugonie, JHEP 0507 (2005) 041.

[23] Z. Kunszt, S. Moretti and W.J. Stirling, Z. Phys. C 74 (1997) 479.

[24] A. Djouadi, hep-ph/0503173.

[25] V. Buscher and K. Jacobs, Int. J. Mod. Phys. A 20 (2005) 2523.

[26] ATLAS Collaboration, Technical Proposal CERN/LHCC/94-43, LHCC/P2, 15 December 1994.

[27] S. Munir, talk given at the 'International School of Subnuclear Physics, 43rd Course', Erice, Italy, August 29 - Sept. 7, 2005, to be published in the proceedings, preprint SHEP-05-37, October 2005.

[28] U. Ellwanger and C. Hugonie, hep-ph/0508022.

[29] G. Belanger, F. Boudjema and S. Moretti, in preparation.

[30] S. Moretti, K. Odagiri, P. Richardson, M. H. Seymour and B. R. Webber, JHEP 0204 (2002) 028; G. Corcella et al., JHEP 0101 (2001) 010.

[31] S. Moretti and S. Munir, in preparation. 


\begin{tabular}{|c|ll|c|}
\hline Higgs Flavor & \multicolumn{2}{|c|}{ Points Visible } & Percentage \\
\hline \hline \multirow{5}{*}{$h_{1}$} & Total: & 1345884 & 99.7468 \\
& Alone: & 1345199 & 99.6961 \\
& With $h_{2}:$ & 528 & 0.0391 \\
& With $a_{1}:$ & 152 & 0.0113 \\
& With $h_{2}$ and $a_{1}:$ & 5 & 0.0004 \\
\hline \multirow{5}{*}{$h_{2}$} & Total: & 1253 & 0.0929 \\
& Alone: & 717 & 0.0531 \\
& With $h_{1}:$ & 528 & 0.0391 \\
& With $a_{1}:$ & 3 & 0.0002 \\
& With $a_{1}$ and $a_{1}:$ & 5 & 0.0004 \\
\hline$h_{3}$ & Total: & 0 & 0 \\
\hline \multirow{5}{*}{$a_{1}$} & Total: & 165 & 0.0122 \\
& Alone: & 5 & 0.0004 \\
& With $h_{1}:$ & 152 & 0.0113 \\
& With $h_{2}:$ & 3 & 0.0002 \\
& With $h_{1}$ and $h_{2}:$ & 5 & 0.0004 \\
\hline$a_{2}$ & Total: & 0 & 0 \\
\hline
\end{tabular}

Table 1: Higgs events potentially visible at the LHC through the $\gamma \gamma$ decay mode (i.e., those yielding cross-section time BR rates of order $10 \mathrm{fb}$ or upwards). Percentage refers to the portion of NMSSM parameter space involved for each discovery scenario. 

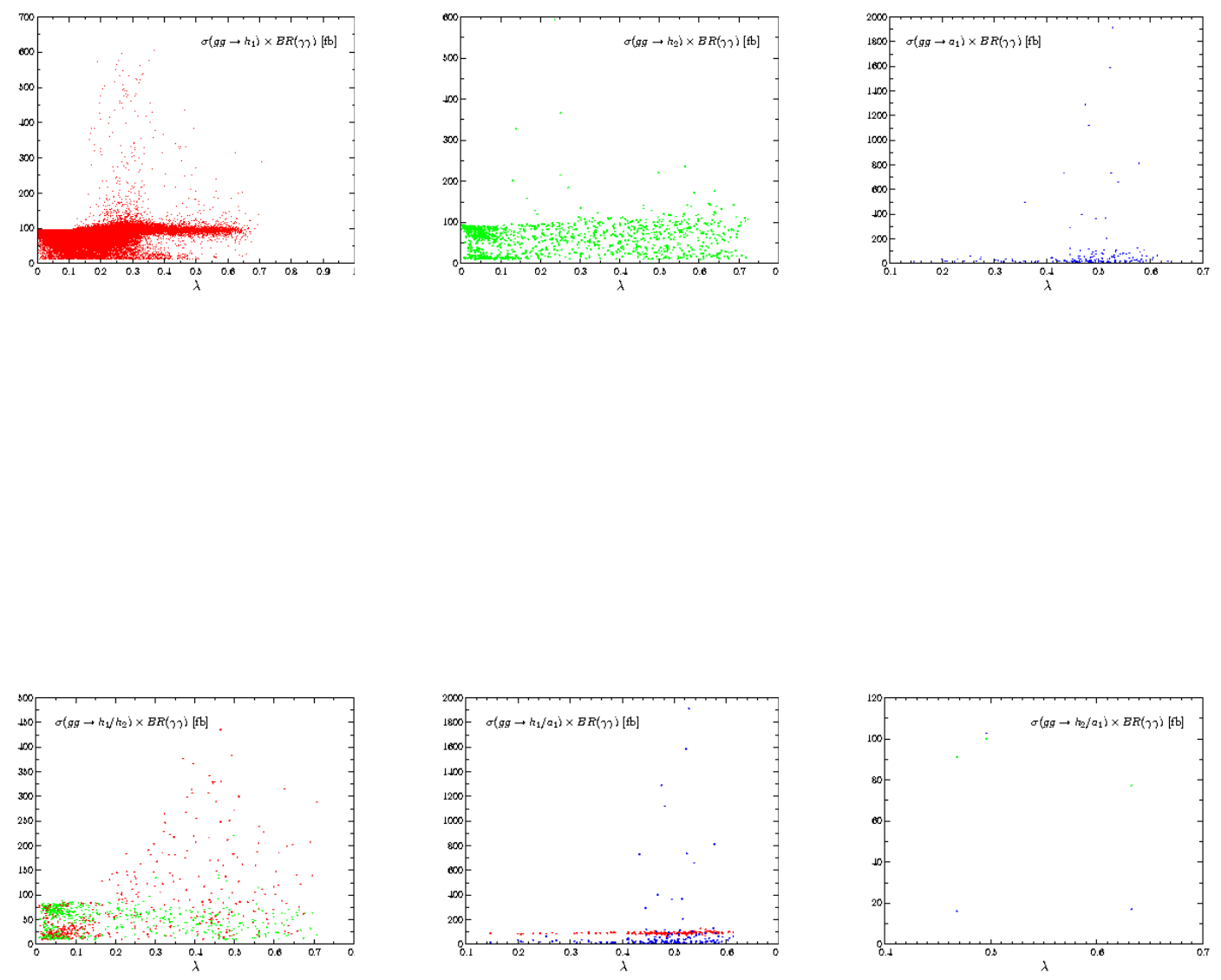

Figure 1: Cross-section times $\mathrm{BR}$ of $h_{1}$ (red), $h_{2}$ (green) and $a_{1}$ (blue), when potentially visible individually and when two of these are potentially visible simultaneously, plotted against the parameter $\lambda$. 

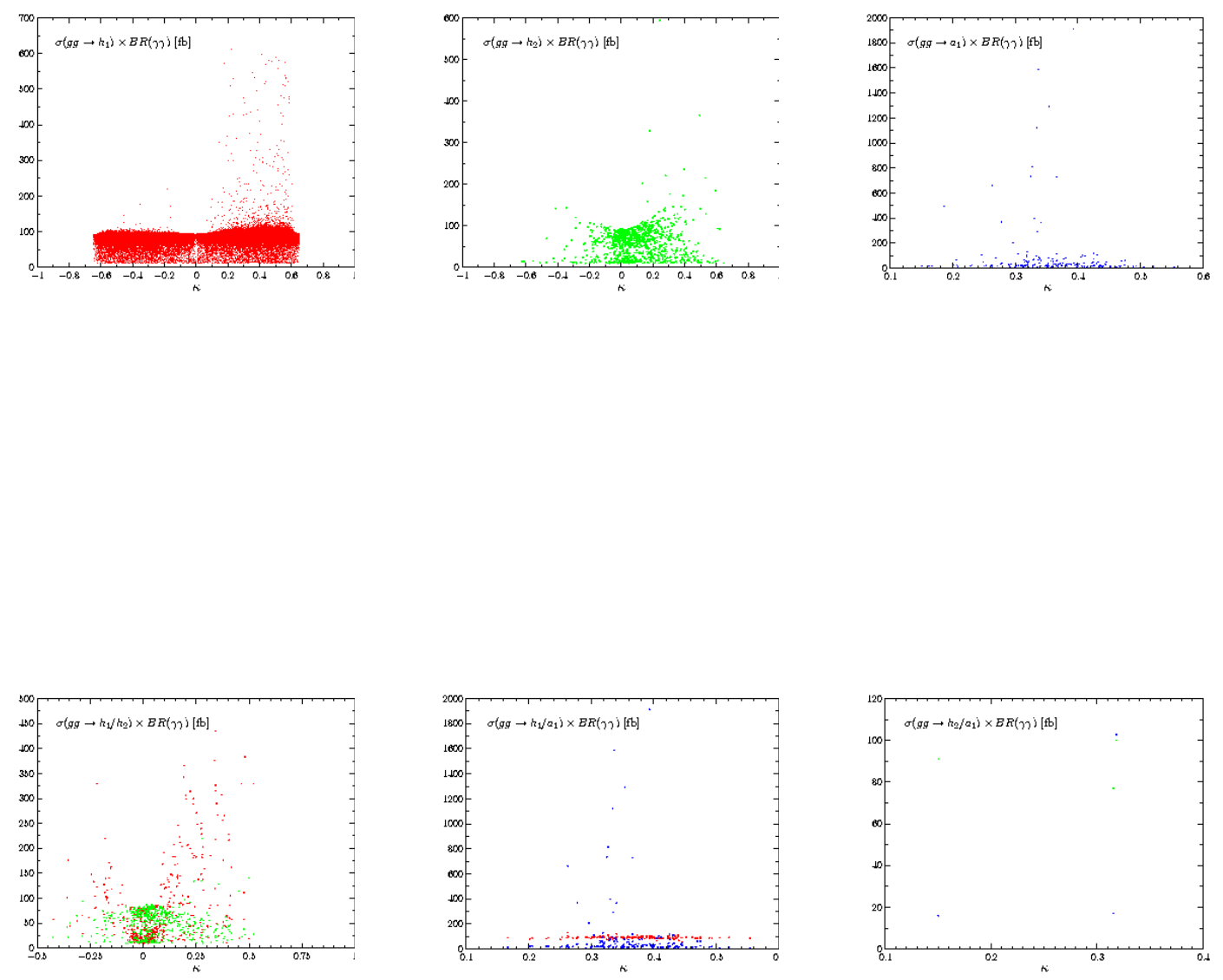

Figure 2: Cross-section times BR of $h_{1}$ (red), $h_{2}$ (green) and $a_{1}$ (blue), when potentially visible individually and when two of these are potentially visible simultaneously, plotted against the parameter $\kappa$. 

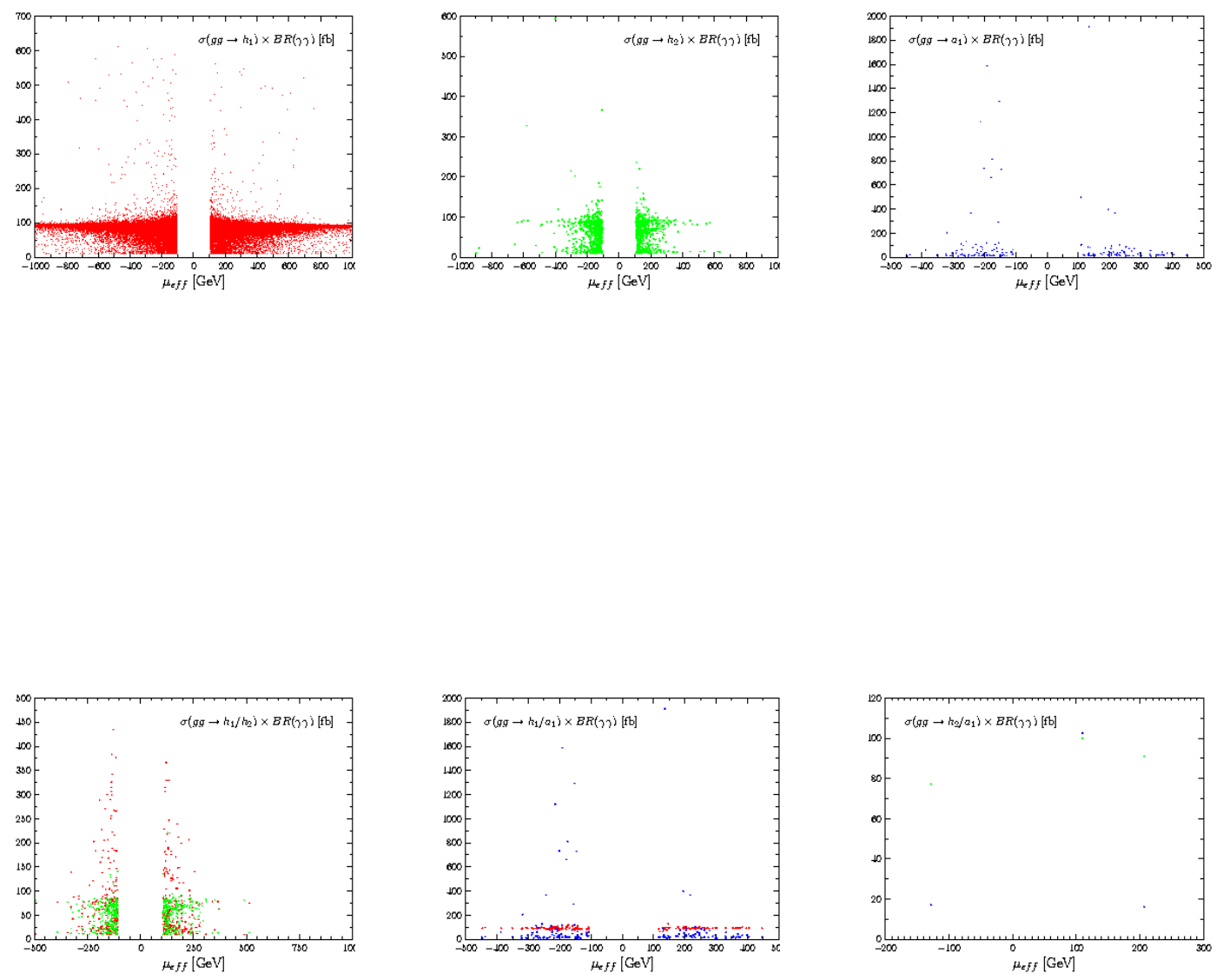

Figure 3: Cross-section times BR of $h_{1}$ (red), $h_{2}$ (green) and $a_{1}$ (blue), when potentially visible individually and when two of these are potentially visible simultaneously, plotted against the parameter $\mu$. 

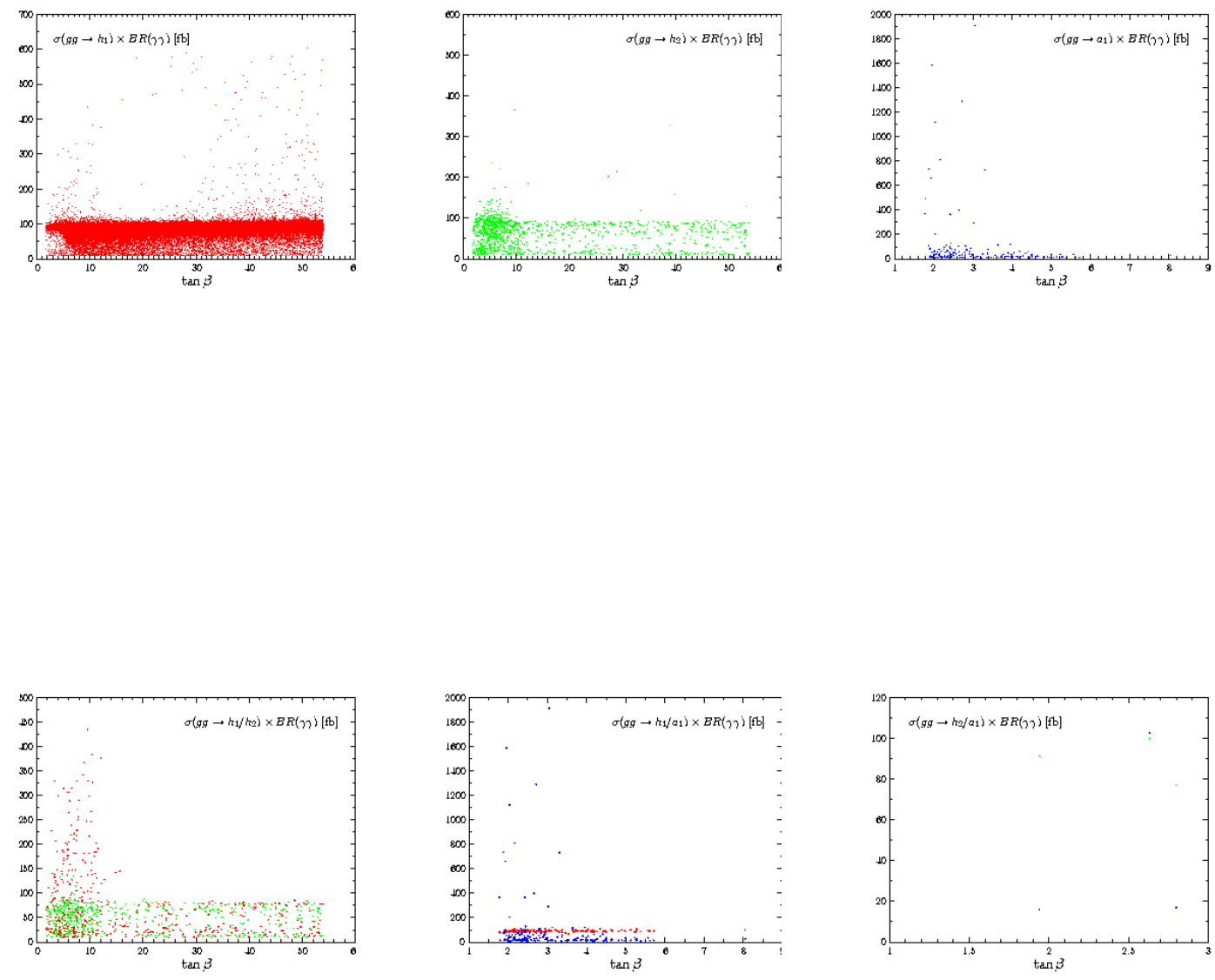

Figure 4: Cross-section times BR of $h_{1}$ (red), $h_{2}$ (green) and $a_{1}$ (blue), when potentially visible individually and when two of these are potentially visible simultaneously, plotted against the parameter $\tan \beta$. 

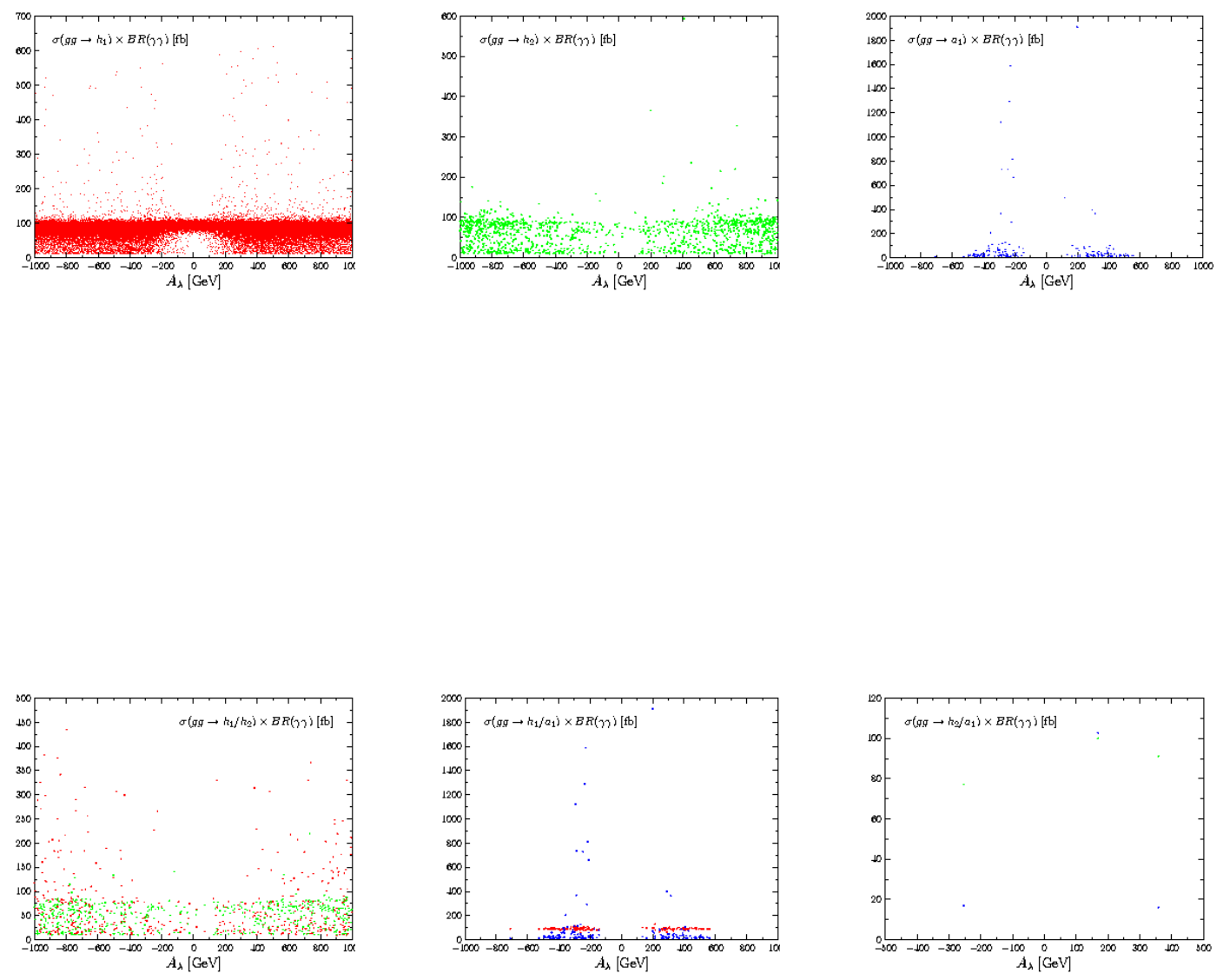

Figure 5: Cross-section times BR of $h_{1}$ (red), $h_{2}$ (green) and $a_{1}$ (blue), when potentially visible individually and when two of these are potentially visible simultaneously, plotted against the parameter $A_{\lambda}$. 

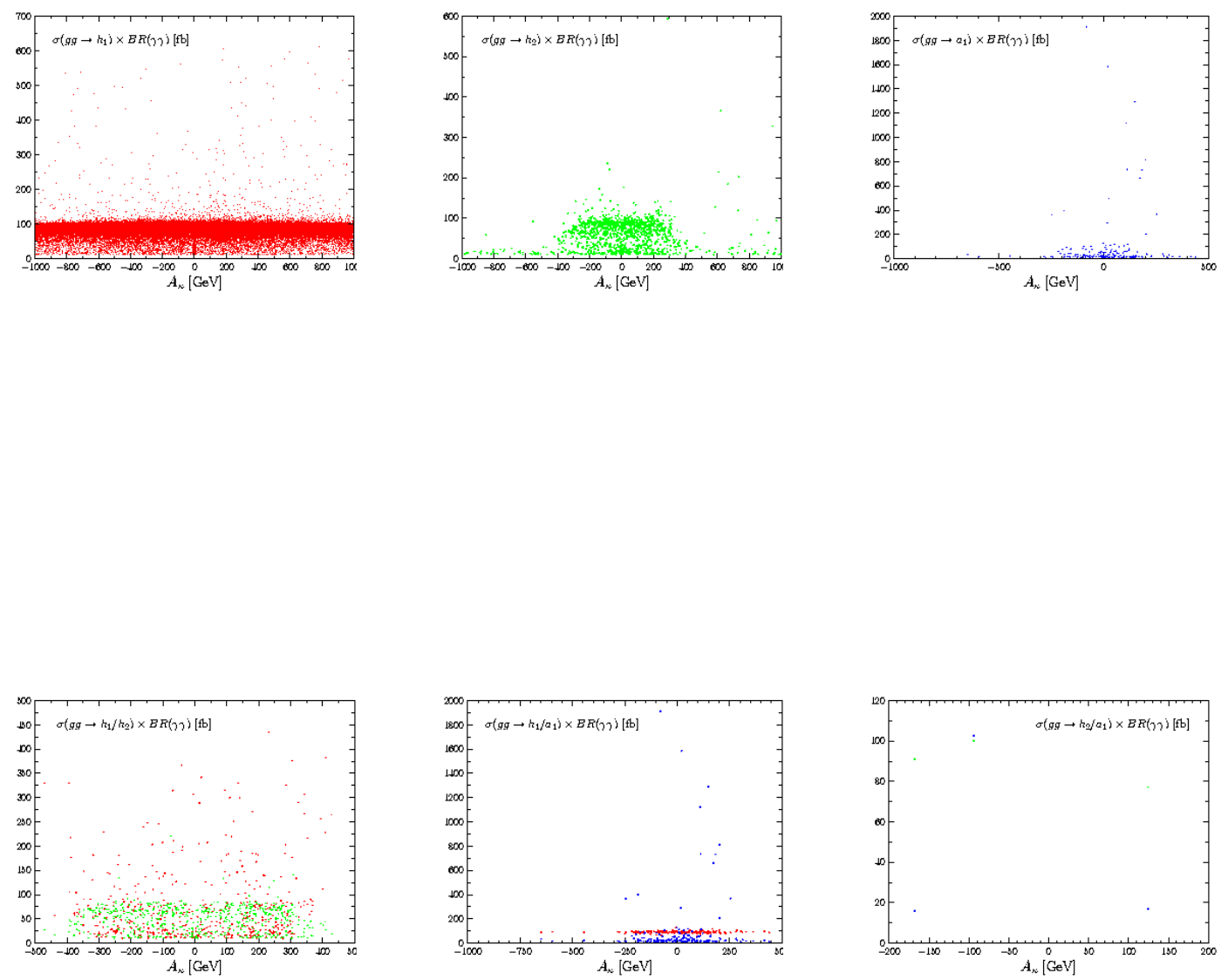

Figure 6: Cross-section times BR of $h_{1}$ (red), $h_{2}$ (green) and $a_{1}$ (blue), when potentially visible individually and when two of these are potentially visible simultaneously, plotted against the parameter $A_{\kappa}$. 

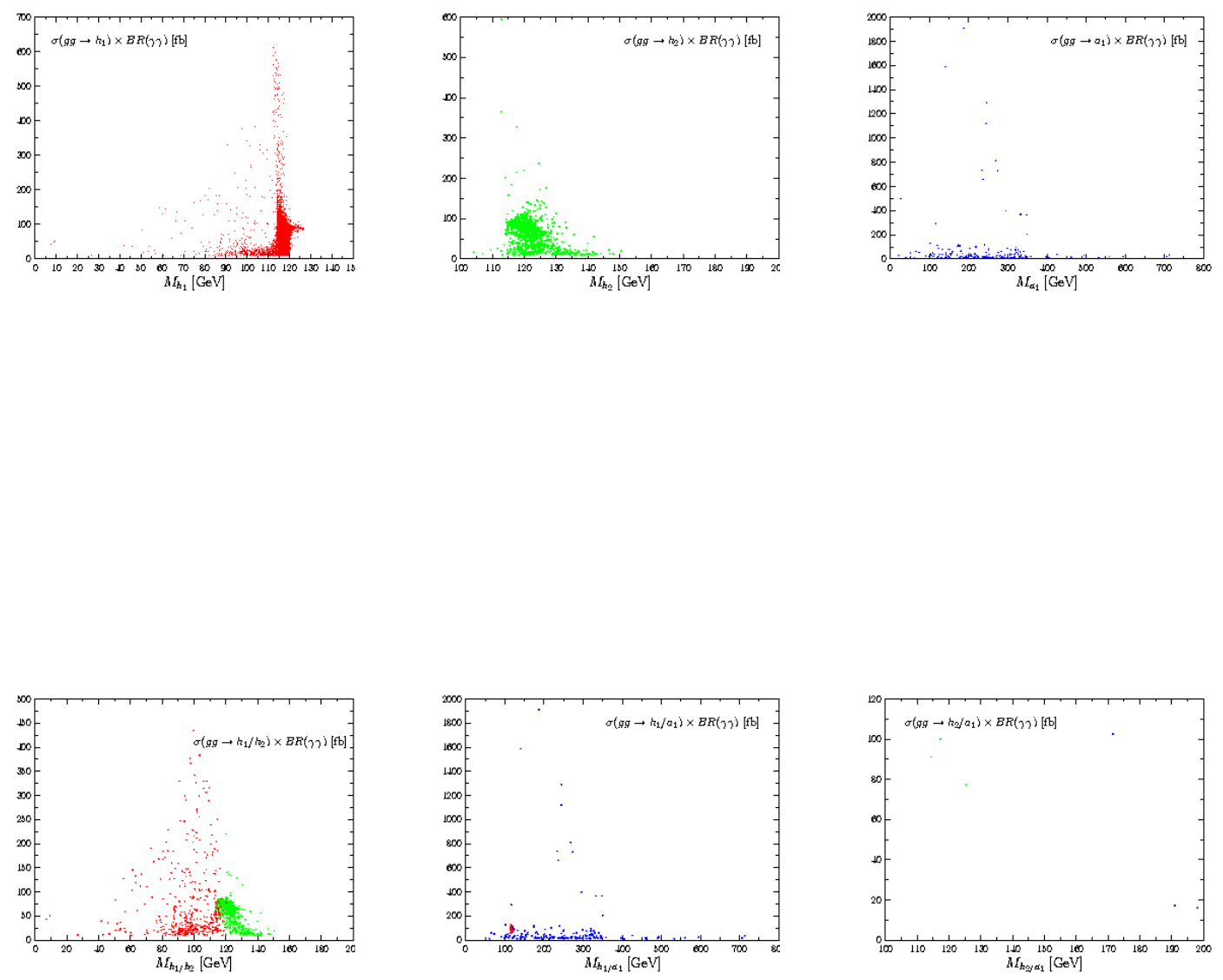

Figure 7: Cross-section times BR of $h_{1}$ (red), $h_{2}$ (green) and $a_{1}$ (blue), when potentially visible individually and when two of these are potentially visible simultaneously, plotted against their respective masses. 


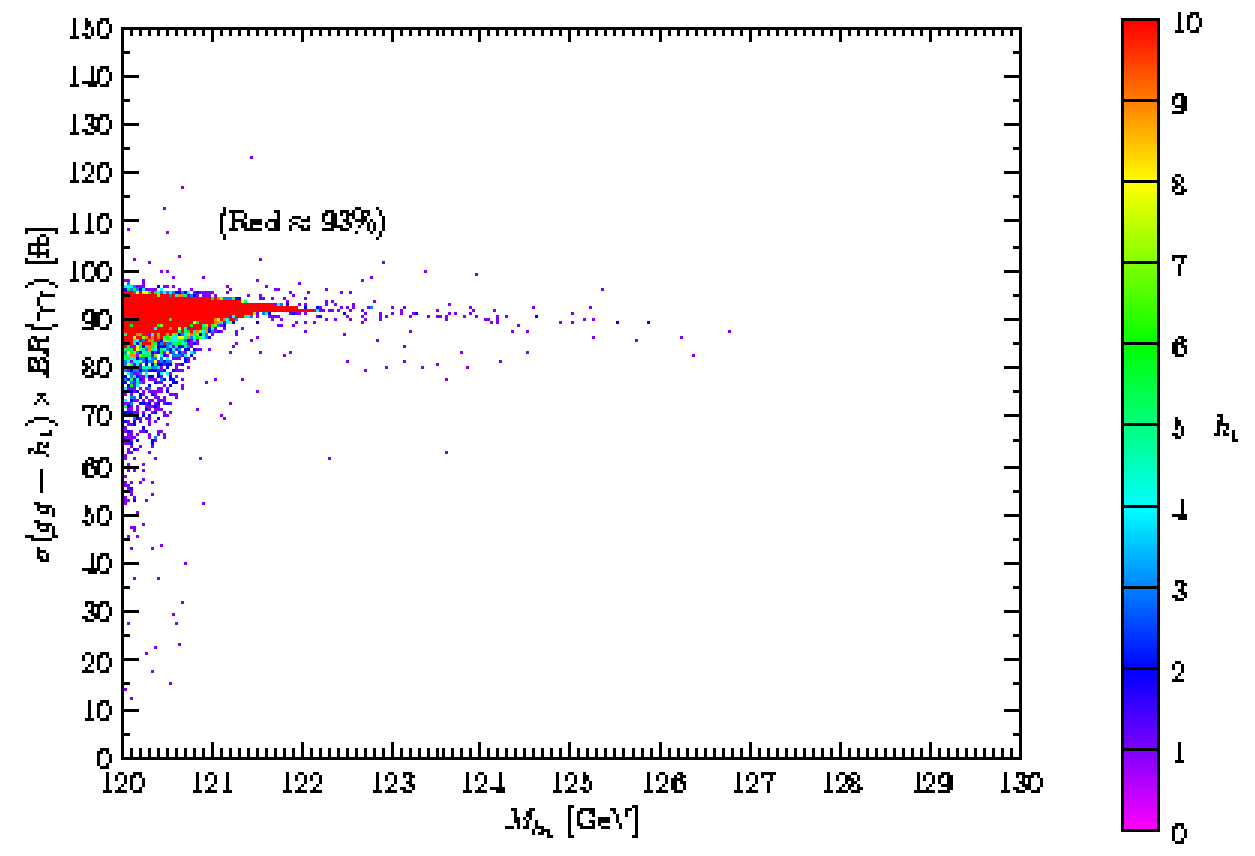

Figure 8: The distribution of points where one has only one potentially visible NMSSM $h_{1}$ state with mass beyond the MSSM upper mass limit on the corresponding Higgs state. The scale on the right represents a measure of density of the points. 

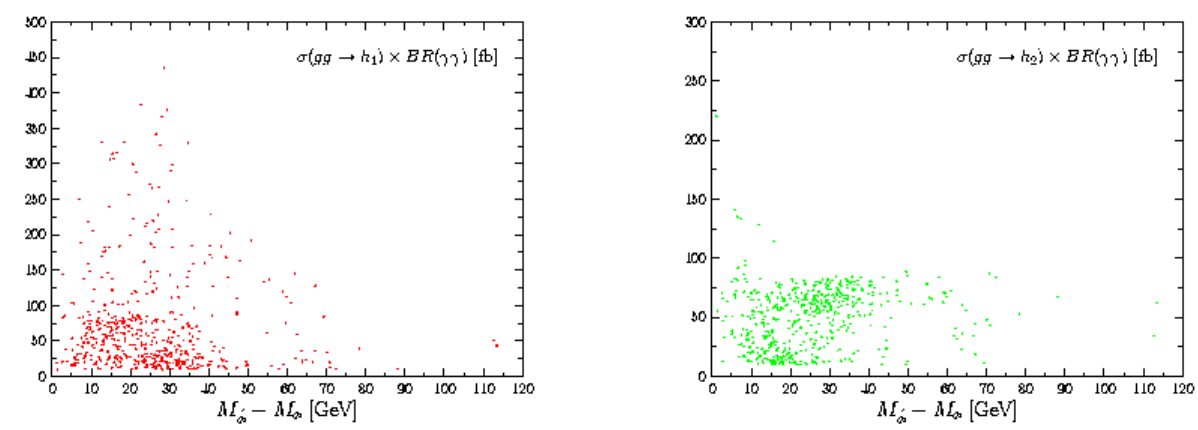

Figure 9: Cross-section times $\mathrm{BR}$ of $h_{1}$ (red) and $h_{2}$ (green) plotted against their mass differences when the two are potentially visible simultaneously. 

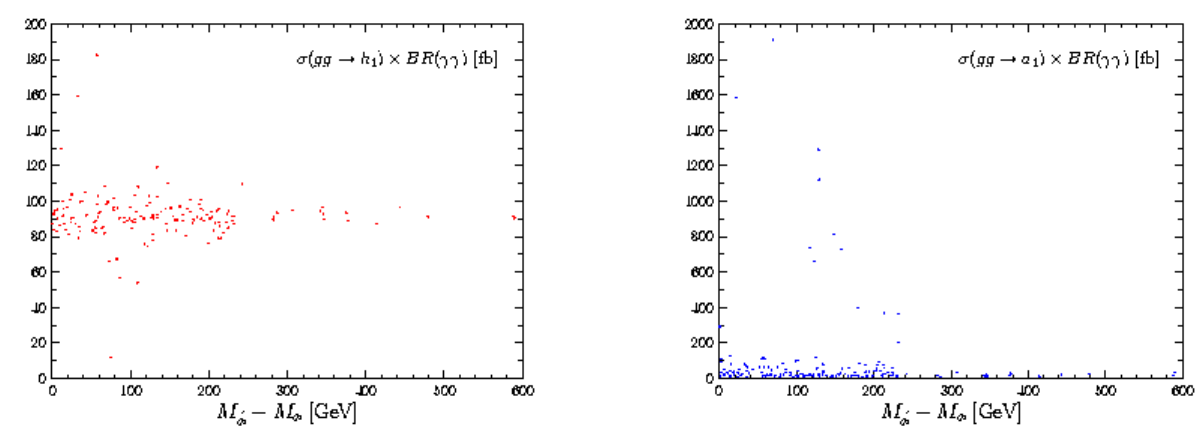

Figure 10: Cross-section times $\mathrm{BR}$ of $h_{1}$ (red) and $a_{1}$ (blue) plotted against thier mass differences when the two are potentially visible simultaneously. 

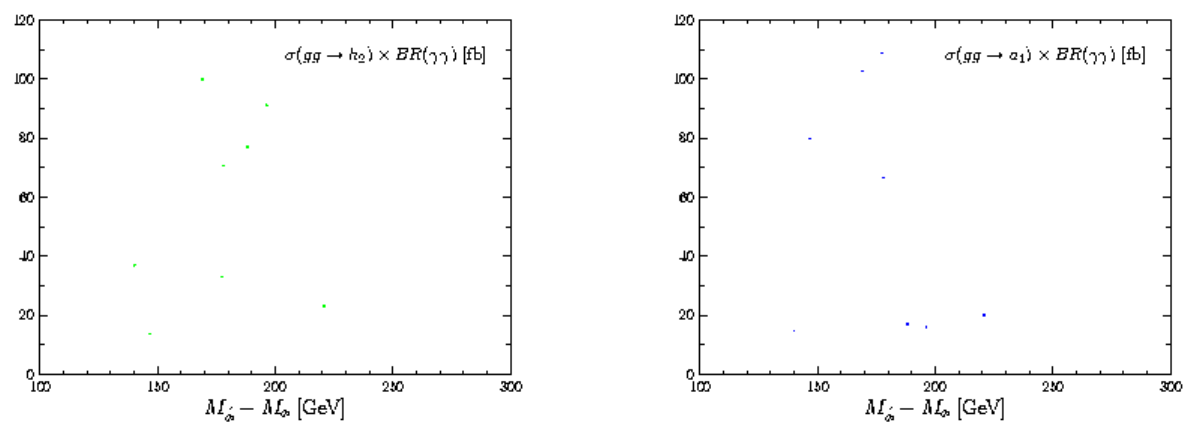

Figure 11: Cross-section times BR of $h_{2}$ (green) and $a_{1}$ (blue) plotted against their mass differences when the two are potentially visible simultaneously. 

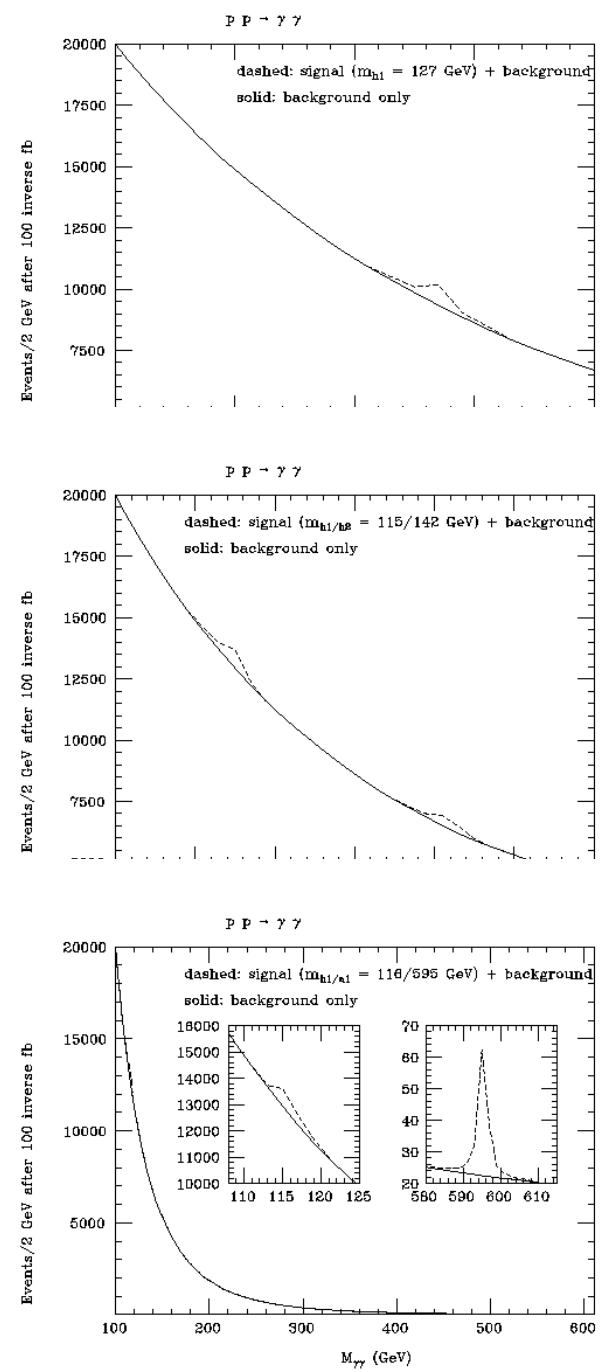

Figure 12: The differential distribution in invariant mass of the di-photon pair after the cuts in $p_{T}^{\gamma}$ and $\eta^{\gamma}$ mentioned in the text, for $100 \mathrm{fb}^{-1}$ of luminosity, in the case of the background (solid) and the sum of signal and background (dashed), for the example points 1.-3. described in the text (from top to bottom, in correspondence). 